\title{
Il reporting di trial randomizzati controllati. Parte 2
}

\section{Francesco Burrai}

\author{
Professore a contratto, Università di Bologna, Bologna
}

\begin{abstract}
The reporting of randomized controlled trials. Part II
Abstract. In our previous article, published on this journal, we analyzed the structure of an RCT in the sections of the title, abstract, introduction, objectives or hypotheses, methods, design, patients, settings, interventions, outcomes, sample size, randomization, and statistical methods. In this second article we will conclude our analysis on RCT reporting with the results section: the flow chart of the patients recruited, dates and recruitment period, demographic and clinical characteristics, results for the outcome, and results for the adverse effects and reactions. In the discussion section: the limits of the trial, generalizability of the results, comparison of the results, registration of the trail and, finally, the financial aspect of the trial.
\end{abstract}

Key words: Randomized Controlled Trial, Research, Reporting

Conflict of interest: None.

Financial support: None.

Accettato: 12 Settembre 2014

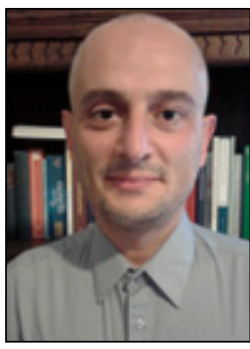

Francesco Burrai

\section{Introduzione}

Come evidenziato nel primo articolo, la valutazione oggettiva dell'efficacia di un intervento assistenziale e sanitario è rappresentato da un gold standard: il trial controllato randomizzato (RCT).

Nel precedente lavoro si è proceduto nell'analisi dei primi nove steps fondamentali che un Autore dovrebbe rispettare per disegnare e condurre in maniera rigorosa ed etica un RCT, ovvero la corretta strutturazione del titolo, dell'abstract, dell'introduzione, gli obiettivi o ipotesi, i metodi, il disegno, i pazienti, il setting, gli interventi, gli outcome, la dimensione del campione, la randomizzazione e i metodi statistici. In questo secondo lavoro analizzeremo i successivi steps di un RCT, ovvero per la sezione dei Risultati la flow chart del flusso dei pazienti, le date e il periodo di reclutamento, le caratteristiche demografiche e cliniche, i risultati per gli outcome, i risultati per gli effetti indesiderati e avversi. Nella sezione Discussione: i limiti di un trial, la generalizzabilità dei risultati, il confronto dei risultati, la registrazione del trial e infine il finanziamento di un trial.

\section{Risultati}

Primo step da includere nell'esposizione dei risultati è la descrizione del flusso dei partecipanti. Il flusso dei pazienti deve essere presentato attraverso l'uso di una flow chart. La flow chart mostra chiaramente tutte le fasi dell'RCT, indicando in quale fasi di studio e temporale si trovano i pazienti. Le fasi sono rappresentate da:

1. Arruolamento, composto dal numero dei pazienti sottoposti a valutazione per l'eleggibilità; dal numero totale dei pazienti non eleggibili e motivazioni della non eleggibilità con relativa numerosità; dal numero dei pazienti sottoposti a randomizzazione.

2. Allocazione, composta dal numero dei pazienti allocati ai gruppi paralleli e sottoposti al trattamento, e dal numero e motivazione dell'eventuale non-trattamento nei due gruppi.

3. Follow-up, composto dal numero dei pazienti persi e relative motivazioni e dal numero dei pazienti che hanno sospeso l'intervento e loro numerosità.

4. Analisi, composta dal numero dei pazienti inclusi nell'analisi statistica e dal numero dei pazienti che non sono stati sottoposti ad analisi con relative motivazioni.

Punti critici nella trasparenza dei trial longitudinali sono:

a) Indicare il numero dei partecipanti eleggibili. Questo permette una analisi critica sulla rappresentatività di tutti i partecipanti al trial per la validità esterna (1).

b) Indicare chiaramente il numero e le motivazioni della perdita dei pazienti nel follow-up $(2,3)$, o esclusi dopo la randomizzazione, il perché non sono stati trattati o sono stati esclusi dalle analisi (4).

c) Indicare chiaramente se sono stati inclusi nelle analisi tutti 
i pazienti randomizzati, presenti nel gruppo di allocazione originale (analisi intention to treat). L'omissione o la falsificazione di tali dati, introduce dei bias, soprattutto nell'affermazione di conclusioni errate.

\section{Date e periodo di reclutamento}

Importante anche riportare nel trial quando è stato condotto lo studio, quale è stato il periodo di reclutamento e la sua durata, la durata del follow-up, la durata dello studio. Questi dati permettono di contestualizzare il periodo storico del trial, utile per compararlo ad altri trial simili e per capire, per esempio, la velocità di reclutamento dei pazienti.

\section{Le caratteristiche demografiche e cliniche}

I trial devono presentare una Tabella in cui vengono riportati le caratteristiche demografiche e cliniche dei pazienti per $\mathrm{i}$ gruppi. Il confronto tra $\mathrm{i}$ due gruppi permette di valutare la comparabilità, la rilevanza, l'applicabilità dei risultati. A volte i gruppi presentano differenze di omogeneità, le quali non sono imputabili a bias di selezione per scorretta randomizzazione, ma alle fluttuazioni casuali nel campione randomizzato. I dati demografici e clinici dei gruppi devono essere presentati in questo modo:

1) indici di tendenza centrale come la media, moda e mediana;

2) indici di dispersione come la deviazione standard e range;

3) indici di distribuzione come frequenze e percentuali.

\section{I risultati per gli outcome}

Per ogni outcome in ogni gruppo indicare il valore dell'outcome (per esempio: frequenze, percentuali, medie con DS), i limiti confidenza al 95\% per indicare l'incertezza della stima (5), e il p-value per indicare la significatività statistica. La trasparenza dei trial richiede che vengano mostrati tutti i risultati per tutti gli outcome nei gruppi, e mai presentare solo i risultati significativi e mai presentarli solo con i valori del p-value $(6,7)$.

\section{I risultati per gli effetti indesiderati e avversi}

Il trial dovrebbe presentare: 1) un elenco degli effetti avversi e la loro specificazione, anche in riferimento alla bibliografia esistente; 2) specificare il rischio assoluto per ogni evento avverso; 3 ) indicare il numero dei pazienti che hanno mostrato gli effetti avversi; 4) nella Discussione, analizzare gli aspetti dei rischi e benefici del trattamento. La presenza di questi dati permette al lettore di capire se gli interventi possono essere utili e accettabili, influenzando le decisioni sul loro utilizzo.

\section{Discussione}

\section{I limiti di un trial}

L'omissione di eventuali limiti presenti in un trial, non permette di individuare eventuali bias e dunque i risultati presentati possono essere erroneamente interpretati. I limiti spesso sono dovuti all'utilizzo di campioni di piccole dimensioni, studi monocentrici, omissioni di bias, imprecisioni nell'utilizzo de- gli strumenti di misura degli outcome, come scale non idonee all'outcome, scale non validate, valutatore non formato. Un importante limite è la non indicazione dei limiti di confidenza, ma solo indicazione dei p-value, il che produce una distorsione nell'interpretazione dei risultati. Se un trial afferma che un risultato è statisticamente non significativo in base al p-value, senza indicare i limiti di confidenza, gli Autori traggono la conclusione che non esistono differenze clinicamente significative, ovvero gli interventi sono equivalenti tra i due gruppi. Invece, la presenza dei limiti di confidenza dà informazioni fondamentali sulla rilevanza clinica di un intervento, indipendentemente dal valore di p. Gli Autori dovrebbero sempre tenere presente che esiste una differenza tra rilevanza clinica e significatività statistica.

\section{La generalizzabilità dei risultati}

Il concetto di generalizzabilità è legato alla proprietà che hanno i risultati del trial di essere applicati o generalizzati ad altre popolazioni, dunque al grado di validità esterna. Per esempio, potremmo chiederci se i risultati del trial possono essere attesi anche in altri tipi di setting assistenziali, oppure se i risultati possono essere generalizzati ad un solo tipo di paziente, o a popolazioni diverse rispetto, per esempio, all'età, al grado di autosufficienza.

La validità esterna però è legata alla qualità della validità interna, la quale ne è prerequisito. Un trial che non ha una capacità di evitare bias nelle fasi di pianificazione, conduzione e analisi, determina risultati non validi, dunque la validità esterna non è assolutamente garantita.

Fondamentale appare allora la trasparenza e la chiarezza nel definire correttamente il tipo di paziente, le tempistiche di reclutamento, i criteri di inclusione, il setting assistenziale, dove è stato condotto, quali sono gli interventi messi a confronto, gli outcome, il timing del follow-up, la descrizione precisa di ogni fase della flow chart del trial $(8,9)$.

\section{Il confronto dei risultati}

Infine è necessario confrontare i risultati ottenuti dal trial con quelli provenienti da altri trial ottenuti dalla revisione della letteratura $(10,11)$.

Una revisione sistematica permette di confrontare, per esempio, se i pazienti arruolati e $\mathrm{i}$ risultati sono simili in altri trial.

\section{La registrazione del trial}

Un trial può essere registrato, per esempio, sul sito ClinicalTrials.gov prima dell'arruolamento dei pazienti. Un trial registrato permette di pubblicizzare, rendere trasparente diversi dati fondamentali del trial, utili alla comunità scientifica (12) e rappresenta un dovere etico e morale, perché contrasta il fenomeno di una non-comunicazione di dati. Gli Autori che hanno registrato il loro trial, ottengono un numero univoco di registrazione che deve essere indicato insieme al nome del Registro nel testo dell'articolo. Registrare un trial permette alla comunità scientifica di conoscere il protocollo, che è lo 
strumento metodologico fondamentale di un RCT, evitando così eventuali problematiche dovute al reporting selettivo o a variazioni non dichiarate nel trial. Il protocollo può essere pubblicizzato, per esempio, facendolo pubblicare da riviste tipo Trials, o nei siti della rivista stessa o in quello dei ricercatori.

\section{Il finanziamento di un trial}

Trial che ricevono finanziamento, possono presentare un rischio di influenza da parte dell'ente finanziatore per esempio sul disegno del trial, sulla sua conduzione, sull'analisi e reporting. Trial che presentano finanziamenti da case farmaceutiche sono a rischio di produrre risultati a favore del farmaco dell'azienda produttrice (13-15). Fondamentale riportare nel trial le fonti di finanziamento, il ruolo nel trial e l'assenza di coinvolgimento.

\section{Conclusioni}

La stragrande maggioranza delle scelte assistenziali si basa su trial. La trasparenza e la correttezza metodologica sono le fonti di serietà, di eticità e moralità di un trial. Chi struttura un trial deve garantire l'assoluta imparzialità nei confronti dei gruppi sottoposti a studio nel trial. L'obiettivo metodologico indispensabile si ritrova nello sforzo costante nel garantire, nel preservare, nel neutralizzare ogni possibile fonte di bias in qualsiasi step del trial. La nascita di un bias, il suo non riconoscimento o manipolazione, può inficiare negativamente la validità interna e di conseguenza la validità esterna di un trial, in poche parole, non è utile alla comunità scientifica, ai pazienti, al progresso scientifico. L'errore di sovrastima dell'efficacia di un intervento è sempre presente nei trial non randomizzati $(16,17)$ e solo l'applicazione corretta di un processo di randomizzazione permette di eliminare gravi bias come quelli di selezione e di confondimento.

Ma anche se è stata eseguita una perfetta randomizzazione, $\mathrm{i}$ bias si possono innescare in ogni altra fase dell'RCT, soprattutto in queste variabili:

a) scarse competenze metodologiche (18);

b) scarse competenze cliniche;

c) impegno non scrupoloso $(19,20)$;

d) reporting scorretti;

e) non trasparenza e non chiarezza;

f) non eticità (21);

g) presenza di outcome soggettivi;

h) occultamento della lista di randomizzazione inadeguato;

i) assenza di blinding.
Gli Autori di RCT dovrebbero acquisire una metodologia corretta, per garantire un adeguato reporting, per contribuire in maniera trasparente e chiara al progresso delle conoscenze, pubblicando trial validi, utili al miglioramento continuo, efficace ed efficiente dell'assistenza delle persone.

\section{Riassunto}

Nel precedente articolo pubblicato su questa rivista, abbiamo analizzato la struttura di un RCT nelle sezioni del titolo, dell'abstract, l'introduzione, gli obiettivi o ipotesi, i metodi, il disegno, i pazienti, il setting, gli interventi, gli outcome, la dimensione del campione, la randomizzazione e i metodi statistici. In questo secondo articolo chiuderemo l'analisi di un reporting di RCT nella sezione dei Risultati: la flow chart del flusso dei pazienti, le date e il periodo di reclutamento, le caratteristiche demografiche e cliniche, i risultati per gli outcome, i risultati per gli effetti indesiderati e avversi. Nella sezione Discussione: i limiti di un trial, la generalizzabilità dei risultati, il confronto dei risultati, la registrazione del trial e infine il finanziamento di un trial.

Parole chiave: Random Control Trial, Ricerca, Reporting

Dichiarazione di conflitto di interessi: L'Autore dichiara di non avere conflitto di interessi.

Contributi economici agli Autori: L'Autore dichiara di non aver ricevuto sponsorizzazioni economiche per la preparazione dell'articolo.

Indirizzo dell'Autore:

Prof. Francesco Burrai

Professore a contratto

Università di Bologna

Viale Ercolani 6

40138 Bologna

francesco.burrai2@unibo.it 


\section{Bibliografia}

1. Meinert CL. Beyond CONSORT: need for improved reporting standards for clinical trials. Consolidated Standards of Reporting Trials. JAMA. 1998; 279(18): 1487-9.

2. Altman DG. Randomisation. BMJ. 1991; 302(6791): 1481-2.

3. Sackett DL, Gent M. Controversy in countingin clinical trials. N Engl J Med. 1979; 301(26): 1410-2.

4. Egger M, Jüni P, Bartlett C. Value of flow diagrams in reports of randomized controlled trials. JAMA. 2001; 285(15): 1996-9.

5. Altman DG. Clinical trials and meta-analisys. In: Altman DG, Machin D, Bryant TN, Gardner MJ, eds Statistics with confidence. $2^{\text {nd }}$ ed. BMJ Books. 2000: 120-38.

6. Gardner MJ, Altman DG. Confidence intervals rather than $p$ values: estimation rather than hypothesis testing. Br Med J. (Clin Res Ed): 1986; 292(6522): 746-50.

7. Bailar JC $3^{\text {rd }}$, Mosteller F. Guidelines for statistical reporting in articles for medical journals. Amplifications and explanations. Ann Intern Med 1988; 108(2): 266-73.

8. King M, Nazareth I, Lampe F, et al. Conceptual framework and systematic review of the effects of participants' and professionals' preferences in randomised controlled trials. Health Technol Assess. 2005; 9(35): 1-186.

9. Djulbegovic B, Lacevic M, Cantor A, et al. The uncertainty principle and industry-sponsored research. Lancet. 2000; 356(9230): 635-8.

10. Sadler LC, Davison T, McCowan LM. A randomised controlled trial and meta-analysis of active management of labour BJOG. 2000; 107(7): 909-15.
11. Gøtzsche PC, Gjørup I, Bonnén H, Brahe NE, Becker U, Burcharth F. Somatostatin versus placebo in bleeding oesophageal varices: randomised trial and meta-analysis. BMJ. 1995; 310(6993): 1495-8.

12. Simes RJ. Publication bias: the case for an international registry of clinical trials. J Clin Oncol. 1986; 4(10): 1529-41.

13. Lexchin J, Bero LA, Djulbegovic B, Clark O. Pharmaceutical industry sponsorship and research outcome and quality: systematic review. BMJ. 2003; 326(7400): 1167-70.

14. Kjaergard LL, Als-Nielsen B. Association between competing interests and authors' conclusions: epidemiological study of randomised clinical trials published in the BMJ. BMJ. 2002; 325(7358): 249.

15. Sismondo S. How pharmaceutical industry funding affects trial outcomes: causal structures and responses. Soc Sci Med. 2008; 66(9):1909-14

16. Deeks JJ, Dinnes J, D'Amico R, et al. Evaluating non-randomised intervention studies. Health Technol Assess. 2003; 7(27): iii-x, 1-173.

17. Kunz R, Vist G, Oxman AD. Randomisation to protect against selection bias in healthcare trials. Cochrane Database Syst Rev. 2007; (2): MR000012.

18. Collins R, MacMahon S. Reliable assessment of the effects of treatment on mortality and major morbidity, I: clinical trials. Lancet. 2001; 357(9253): 373-80.

19. Schulz KF. Randomised trials, human nature, and reporting guidelines. Lancet. 1996; 348(9027): 596-8.

20. Schulz KF. Subverting randomization in controlled trials. JAMA. 1995; 274(18): 1456-8.

21. Murray GD. Promoting good research practice. Stat Methods Med Res. 2000; 9(1): 17-24. 